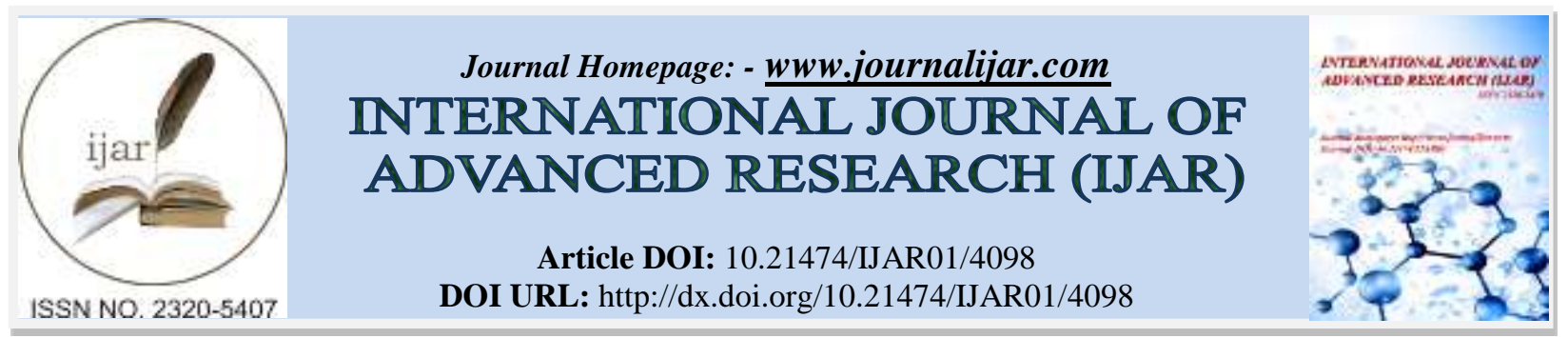

RESEARCH ARTICLE

\title{
ASSESSMENT OF RADIOMORPHOMETRIC INDICES ON DIGITAL PANORAMIC AND CONE BEAM COMPUTED TOMOGRAPHIC IMAGES IN OSTEOPOROTIC EGYPTIAN FEMALES.
}

\author{
Sarah M. Kenawy*1, Dina M. El Beshlawy ${ }^{2}$ and Mushira M. Dahaba ${ }^{3}$ \\ 1. Assistant Lecturer, Oral and Maxillofacial Radiology Department, Faculty of Dentistry, Cairo University, \\ Cairo, Egypt. \\ 2. Associate Professor, Oral and Maxillofacial Radiology Department, Faculty of Dentistry, Cairo University, \\ Cairo, Egypt \\ 3. Professor, Oral and Maxillofacial Radiology Department, Faculty of Dentistry, Cairo University, Cairo, Egypt
}

\section{Manuscript Info}

Manuscript History

Received: 07 March 2017

Final Accepted: 13 April 2017

Published: May 2017

Key words:-

Cone Beam Computed Tomography;

Panoramic Radiography; Dual Energy xray Absorptiometry; Osteoporosis;

Radiomorphometric indices.

\section{Abstract}

Background and purpose: This study aimed to assess the effectiveness of radiomorphometric indices based on digital panoramic and cone beam computed tomography (CBCT) images as osteoporosis predictors in healthy and osteoporotic women. Methods: Twenty selected female volunteers were scanned by digital panoramic, dual Energy x-ray absorptiometry (DEXA) and CBCT scans. According to the DEXA results, they were categorized into either normal or osteopenic/osteoporotic group. The same measurements were performed on digital panoramic, and CBCT images by 3 observers and the average of each was calculated. Results: This study demonstrated that there was no significant correlation between the DEXA results with either panoramic or CBCT indices in the normal, osteopenic and osteoporotic groups. Conclusion: Within the limitation of the current study regarding the limited sample size, the bone of the mandible does not appear to reflect the characteristics of the skeleton as a whole. Panoramic Mandibular Index and Mental Index, with a larger sample size, may serve as prognostic osteoporosis indices.

Copy Right, IJAR, 2016,. All rights reserved.

\section{Introduction:-}

Osteoporosis, the silent epidemic, is defined as a disease characterized by low bone mass and microarchitectural deterioration of bone tissue, leading to enhanced bone fragility and a consequent increase in fracture risk (Nakamoto et al., 2003; Nakamura et al., 2017).

Dual Energy X-ray Absorptiometry (DEXA) is considered the gold standard of methods used to diagnose osteoporosis . This test is capable of measuring bone mineral content at any site in the body but usually is used at central sites (the lumbar spine and the proximal femur) and peripheral sites, including the distal forearm (Kazakia and Majumdar, 2006)

Digital panoramic radiography provides relatively low radiation, time and budget saving, and the ability to supply precise measurements. Recently, panoramic radiography and radiomorphometric indices, which are calculated by 
performing linear measurements on the mandible of the obtained panoramic radiographic images, have attracted clinicians' attention. Medical literature abounds with comparisons of bone mineral density at different skeletal sites with panoramic radiomorphometric indices indicating bone mineral density (BMD) of the mandible (Dutra et al., 2005; Zarch et al., 2011).

Researchers used the computed tomography indices (CTI) on Cone Beam Computed Tomography (CBCT) images to evaluate the mandibular bone quality and examined the potential use of CTI on the CBCT images for the assessment of the BMD in postmenopausal osteoporotic women (Scarfe et al., 2006; Berco et al., 2009; Koh and Kim, 2011).

This study aimed to assess the effectiveness of these indices as osteoporosis predictors in healthy and osteoporotic women.

\section{Patients and Methods:- \\ Subjects:-}

Twenty volunteer females attending the "Out Patients Clinic" of the Faculty of dentistry, Cairo University for routine dental treatment and the "Out Patients Clinic" of Rheumatology Department, Faculty of Medicine, Cairo University were selected and included in the current study after signing an informed written consent. Before performing any procedure, all patients received a thorough explanation concerning the clinical procedures as well as the possible risks and radiation hazards. Performance of this study was approved by the research ethics committee of the Faculty of dentistry, Cairo University.

The participants involved in the current study were selected according to the following criteria: all patients were as possible of the same socioeconomic level, free from any systemic or metabolic disease that could affect bone metabolism and their ages ranging from 40-65 years (mean age of 52.5 years). All patients have both lower premolars bilaterally present and normal bone level or mild bone loss of the premolar area. Females with any systemic disease affecting the bone metabolism, smokers and alcohol consumers, pregnant or suspected to be pregnant, with history of previous vertebral fracture, bone lesions, fractures and deformity or previous mandibular surgery, or with bone loss more than $2 \mathrm{~mm}$ from CEJ at mandibular premolar area, were excluded from the study.

\section{Methods of Examination:-}

All the selected volunteers were subjected to the following diagnostic procedures:

a. Detailed case history and clinical examination: Each of the 20 volunteers was subjected to detailed case history and comprehensive clinical examination of both soft and hard oral tissues for assessing the health status of the oral and maxillofacial region.

b. Digital Panoramic Radiography: Digital panoramic images were obtained for all the 20 volunteers using the "PLANMECAPROLINE XC" Orthopantomograph machine (Planmeca, Helsinki, Finland). The exposure parameters were as follows: $70 \mathrm{kVp}, 12 \mathrm{~mA}$ and $17 \mathrm{sec}$.

c. Dual Energy X-ray Absorptiometry (DEXA): Using the direct digital densitometry of "LUNAR PRODIGY PRIMO DEXA SYSTEM" (GE Healthcare, USA), the BMD and T-score of the lumbar vertebrae (AP spine), the left femoral neck bone as well as the left forearm bones was assessed using the following parameters: 76 $\mathrm{kVp}$ and $3 \mathrm{~mA}$.

\section{Patient Grouping:-}

According to the DEXA analytical results, patients were categorized following the WHO specifications into two groups as follows:

Group I: This group comprised normal individuals ( $\mathrm{n}=10$ patients) with a BMD between -1 and +1 SD (T-score >land <+1).

Group II (A): This group comprised osteopenic patients ( $\mathrm{n}=6$ patients) with a BMD between -1.0 and -2.5 SD below the normal for young healthy adults of the same sex (T-score $<-1.0$ and $>-2.5$ ).

Group II (B): This group comprised osteoporotic patients ( $\mathrm{n}=4$ patients) with a BMD of $-2.5 \mathrm{SD}$ or below (T-score $\leq-2.5)$. 
d. Cone Beam Computed Tomography (CBCT): CBCT images were obtained for all the 20 volunteers using the "I-CAT" (Imaging Sciences International Hatfield, PA, USA) CBCT scanner using the following exposure parameters: kVp: 120, mA: 5, Exposure time: 4 sec, FOV: $13 \times 16 \mathrm{~cm}$, Voxel size: $0.3 \mathrm{~mm}$.

\section{Measurements:-}

A. Panoramic measurements: Using the digital measurement tools of the applied "PlanmecaRomexis viewer" software, all the images were viewed on a 15 inch personal computer, using windows 7 and the images were zoomed until proper vision was obtained. Six indices were obtained from the digital panoramic (1:1) image bilaterally as follows:

\section{The Mental Index (MI):-}

For the MI measurement, the mental foramen was identified. Then a line was plotted perpendicular to another two lines drawn parallel and tangential to the inferior border of the mental foramen and to the inferior border of the mandible corpus. The cortical width at the mental foramen region was measured along this line between these two points of intersection (Klemetti et al., 1994; Ledgerton et al., 1997) (Fig. 1).

\section{The Panoramic Mandibular Index (PMI):-}

For the PMI, the thickness of the inferior border of the mandible below the mental foramen (MI) was measured and divided by the distance from the inferior border of mental foramen to the inferior border of the mandible (Benson et al., 1991) (Fig. 2).

The Alveolar Bone Resorption Degree (M/M Ratio):-

For the M/M ratio, the total alveolar ridge height was measured and then divided by the height from the center of the mental foramen to the inferior border of the mandible (Hirai et al., 1993; Klemetti and Vainio, 1993) (Fig. 3).

\section{The Antegonial Index (AI):-}

The antegonial index is a measurement of cortical width in the region anterior to the gonion at a point identified by extending a line of "best fit" on the anterior border of the ascending ramus down to the lower border of the mandible (Ledgerton et al., 1999) (Fig. 4).

\section{The Gonial Angle (GA):-}

The gonial angle was measured as the angle between a line tangent to the lower border of the mandible and another line tangent to the posterior border of the ramus on each side (Xie and Ainamo, 2004) (Fig. 5).

\section{The Gonial Index (GI):-}

For GI measurements, one line was drawn tangential and parallel to the mandibular ramus and another parallel to the mandibular corpus. A third line starting from the intersection of the first two lines was finally drawn to obtain the measurement for the GI and then the cortical thickness on the line of intersection was measured (Bras et al., 1982) (Fig. 6).

B. CBCT measurements: Using the "i-CATVisionTM" inherent software on the reformatted panoramic views and the coronal cross sectional images, only 3 indices related to the mental foramen (MI, PMI and M/M ratio) were measured on both the default reformatted panoramic cut and the selected coronal cross sectional cut of 0.3 $\mathrm{mm}$ thickness. However, we couldn't measure the AI and GI as the condyle and coronoid of both sides were cut off to adjust the FOV to the mandible only and subsequently minimizing the radiation dose to the study volunteers. In addition, the "i-CATVision ${ }^{\text {TM" }}$ software didn't provide angular measurements; therefore, the gonial angle couldn't be measured. The three indices were obtained bilaterally as follows;

\section{Reformatted Panoramic Images:-}

The measurements of the three indices (MI, PMI\& M/M Ratio) were obtained on the default reformatted panoramic image of each mandible by the same steps followed on the digital panoramic image (Fig. 7).

\section{Cross Sectional Images:-}

The measurements of the same 3 indices were measured on the cross sectional cut which was selected by the means of the orientation lines centralized in the middle of the mental foramen both vertically and horizontally (Fig. 8) 


\section{The Mental Index (MI):-}

For the MI, a line was drawn tangent to the lower border of the mandible and then a line measuring the inferior cortical width was drawn perpendicular to the previous line from the top of the cortex (Fig. 9).

\section{The Panoramic Mandibular Index (PMI):-}

For the PMI, the previous measurement of the inferior cortical width was divided by the measured length of a line drawn from the inferior border of the mental foramen to a line tangent to the lower border of the mandible (Fig. 9).

The Alveolar Bone Resorption Degree (M/M Ratio):-

For the M/M ratio, the total mandibular height was measured and then divided by the height from the center of the mental foramen to the inferior border of the mandible (Both lines reached the tangent to the lower border of the mandible) (Fig. 9).

For each measurement, three observers performed the analysis once. Inter-observer variations were statistically calculated. The means of the trials were pooled and included into further statistical analysis. In an attempt to eliminate the inter-observer errors, not only were the panoramic and CBCT images independently and separately assessed by the three radiologists but they were also performed in a double-blind fashion (none of the observers knew the results of the other and none of them knew any information about the codes of the analyzed images as the radiographed mandibles were coded by one of the staff members at the "Oral and Maxillofacial Radiology Department" who did not share in image analysis.

\section{Statistical Analysis:-}

Data were presented as mean and standard deviation (SD) values. Pearson's correlation coefficient was used to determine significant correlations between different measurements.

Comparison between the three groups regarding BMD data was performed using ANOVA test followed by Tukey's post hoc test for pair-wise comparisons. T-scores data showed nonparametric distribution so, Kruskal-Wallis test was used to compare between the three groups. Mann-Whitney U test was used for pair-wise comparisons between the groups when Kruskal-Wallis test is significant.

Binary logistic regression was used to determine significant predictors of Osteoporosis among different measurements. The dependent variable was the disease (Normal or Disease) while the independent variables were all indices with different modalities. Measurements with the highest scores were considered the most significant predictors of disease. The significance level was set at $\mathrm{P} \leq 0.05$. Statistical analysis was performed with IBM SPSS Statistics Version 20 for Windows.

\section{Results:-}

Correlation between digital panoramic, CBCT measurements and DEXA T-scores:-

There was no statistically significant correlation between digital panoramic, CBCT measurements and T-scores values of Lumbar vertebrae, femur or forearm in all volunteer groups. Except for the osteoporotic group, the PMI measured on digital panorama and MI measured on CBCT cross sectional view where there was a statistically significant negative (inverse) correlation between both of them and T-scores of Lumbar vertebrae (Figs 10 \& 11).

\section{Comparative Study between the Three Groups:- \\ Panoramic measurements:-}

For MI and PMI, there was no statistically significant difference between normal and osteopenic groups; both showed the statistically significantly highest mean MI. Osteoporotic group showed the statistically significantly lowest mean MI. However, for the rest of indices (AI, GI, M/M ratio and GA), there was no statistically significant difference between the three groups.

\section{CBCT Measurements:-}

On the CBCT reformatted panoramic images, there was no statistically significant difference between the three groups considering the MI, PMI and M/M ratio.

For the CBCT cross section view images, considering the MI and PMI, there was no statistically significant difference between normal and osteopenic groups; both showed the statistically significantly highest mean MI. 
Osteoporotic group showed the statistically significantly lowest mean MI. While for the M/M ratio, there was no statistically significant difference between the three groups.

\section{Regression Analysis:-}

Logistic regression model was used to determine the most predictive index for osteoporosis. The dependent variable was the disease status (healthy, osteopenic and osteoporotic) while the independent variables were all indices with different modalities. The results showed that the significant predictors of osteoporosis are the following variables arranged from the highest to lowest scores: PMI (Panoramic), MI (Panoramic), MI (CBCT cross section) and MI (CBCT reformatted panoramic) (Table. 1).

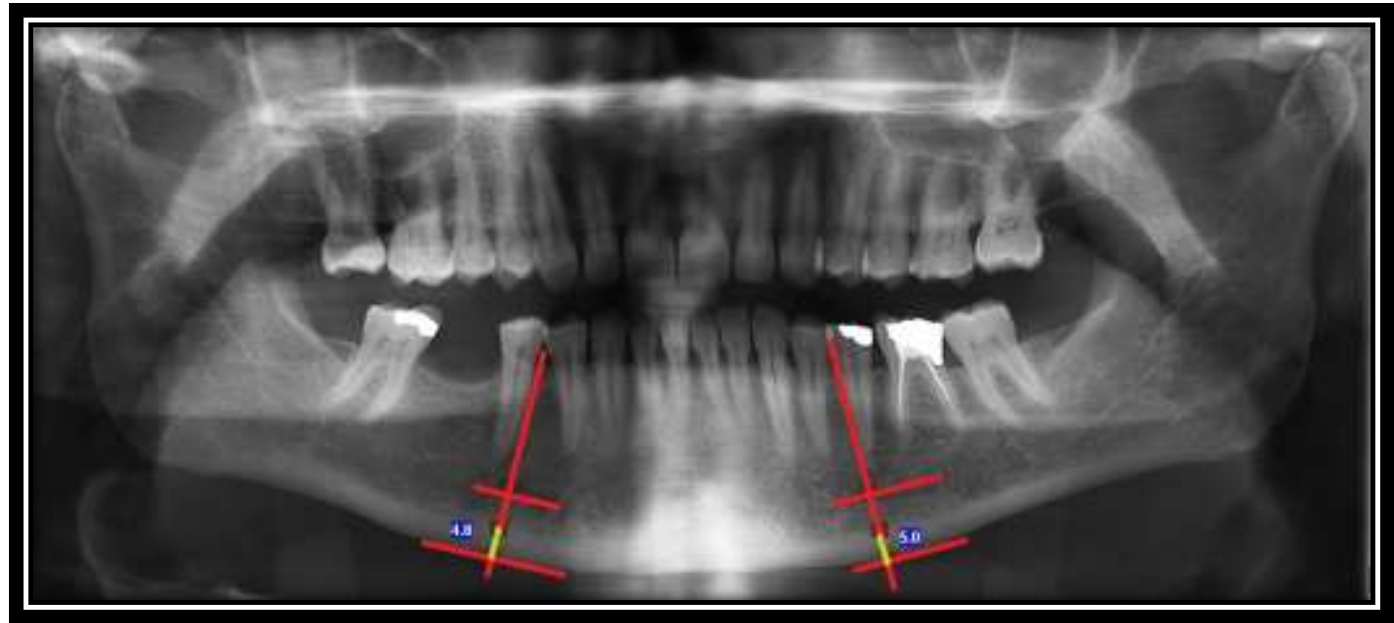

Figure 1: Digital panoramic image showing the measurement of the MI of this case in the right side (4.8 $\mathrm{mm})$ and in the left side $(5 \mathrm{~mm})$.

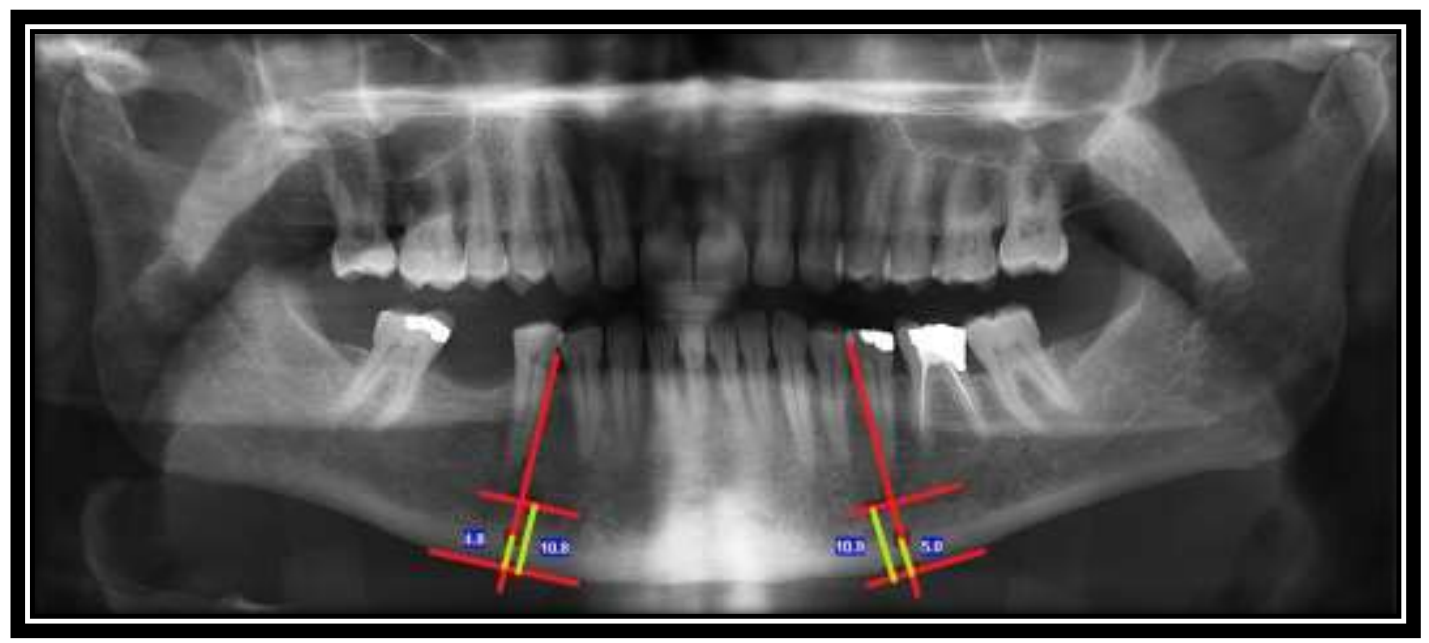

Figure 2: Digital panoramic image showing the measurement of the PMI of this case in the right side $(4.8 \backslash 10.8=$ $0.44)$ and in the left side $(5 \backslash 10.9=0.45)$. 


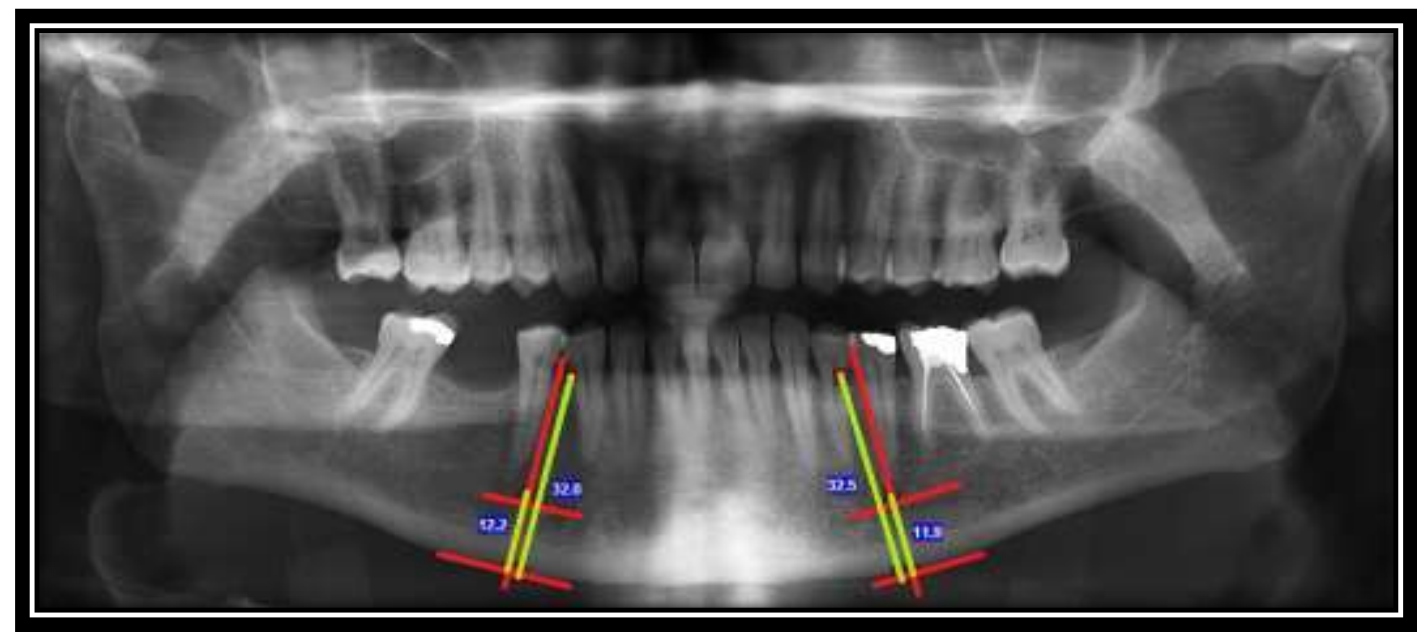

Figure 3: Digital panoramic image showing the measurement of the $\mathrm{M} / \mathrm{M}$ ratio of this case in the right side (32\12.2 $=2.62)$ and in the left side $(32.5 \backslash 11.9=2.73)$.

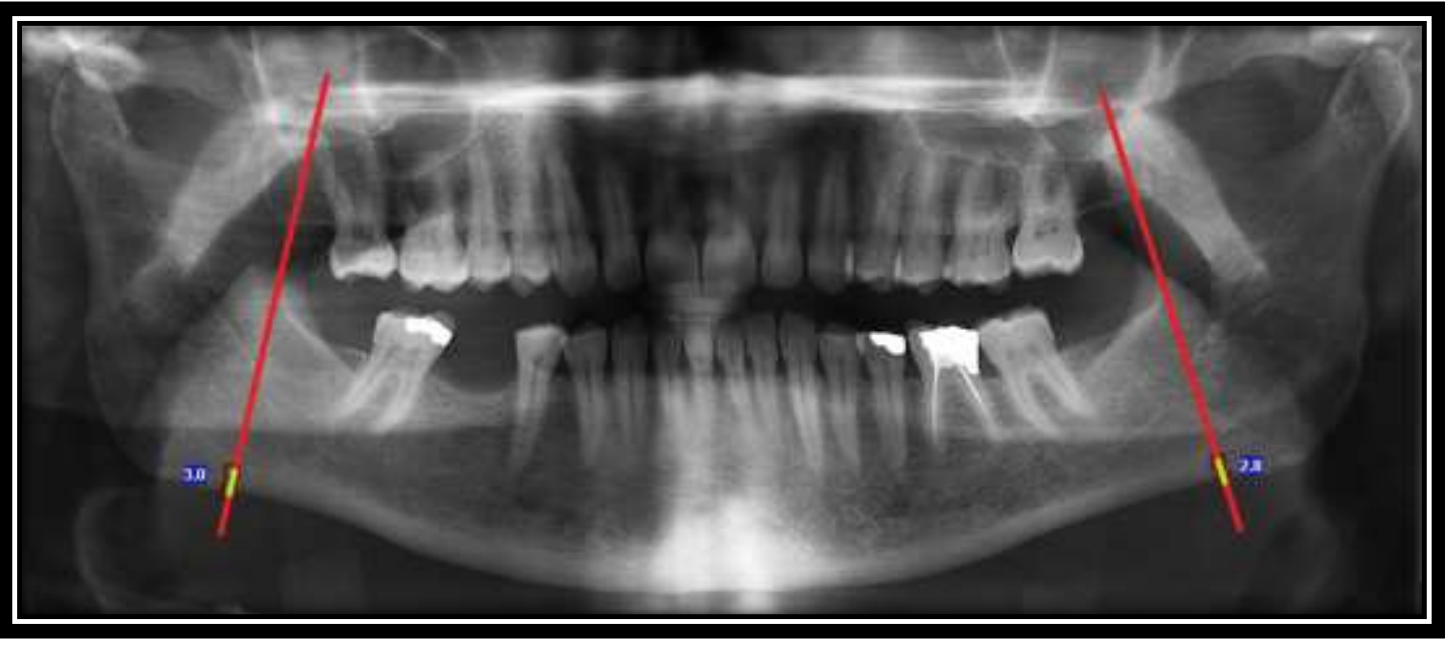

Figure 4: Digital panoramic image showing the measurement of the AI of this case in the right side $(3 \mathrm{~mm})$ and in the left side $(2.8 \mathrm{~mm})$.

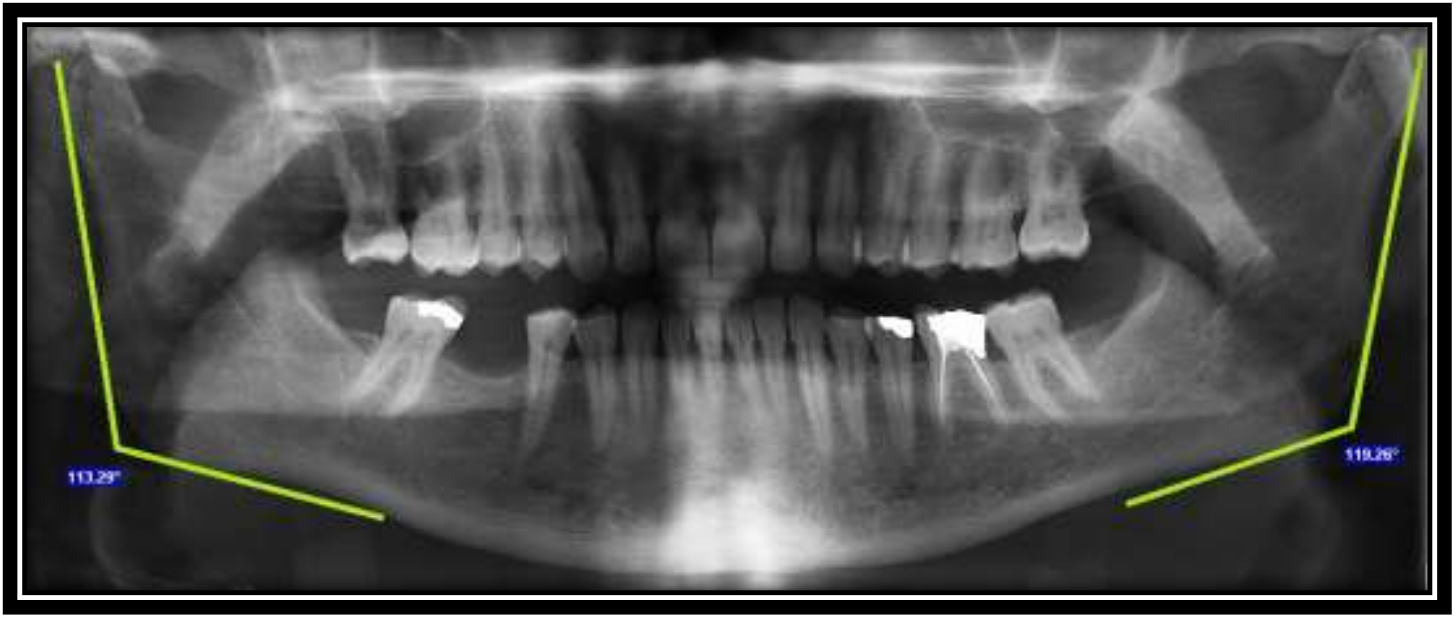

Figure 5: Digital panoramic image showing the measurement of the gonial angle of this case in the right side $\left(113.29^{\circ}\right)$ and in the left side $\left(119.26^{\circ}\right)$. 


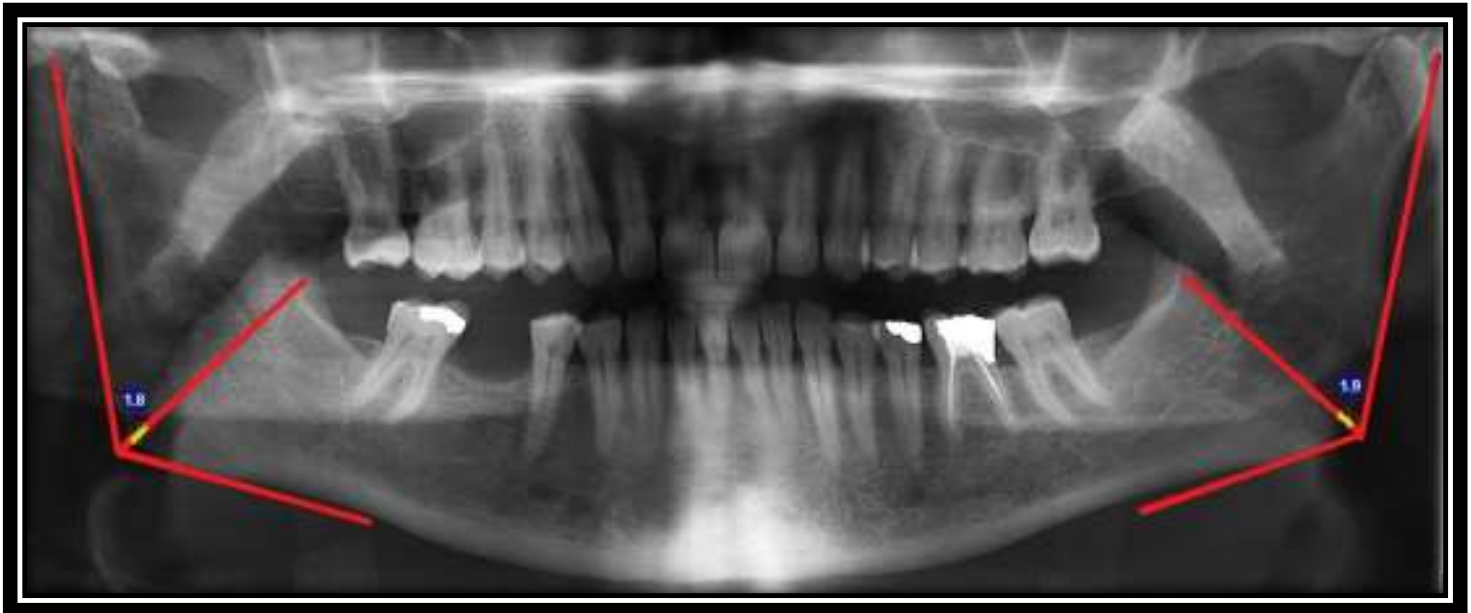

Figure 6: Digital panoramic image showing the measurement of the GI of this case in the right side $(1.8 \mathrm{~mm})$ and in the left side $(1.9 \mathrm{~mm})$.

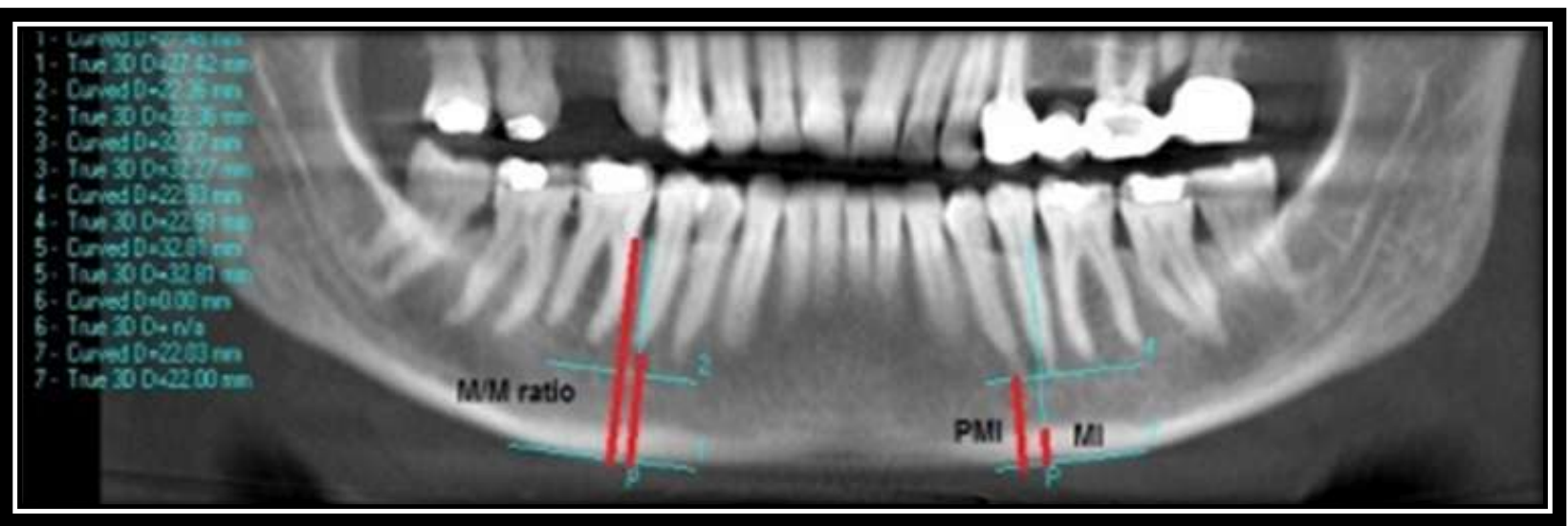

Figure 7: Measurement of the 3 indices (MI, PMI\& M/M ratio) on one of the investigated volunteers on CBCT reformatted panoramic image.

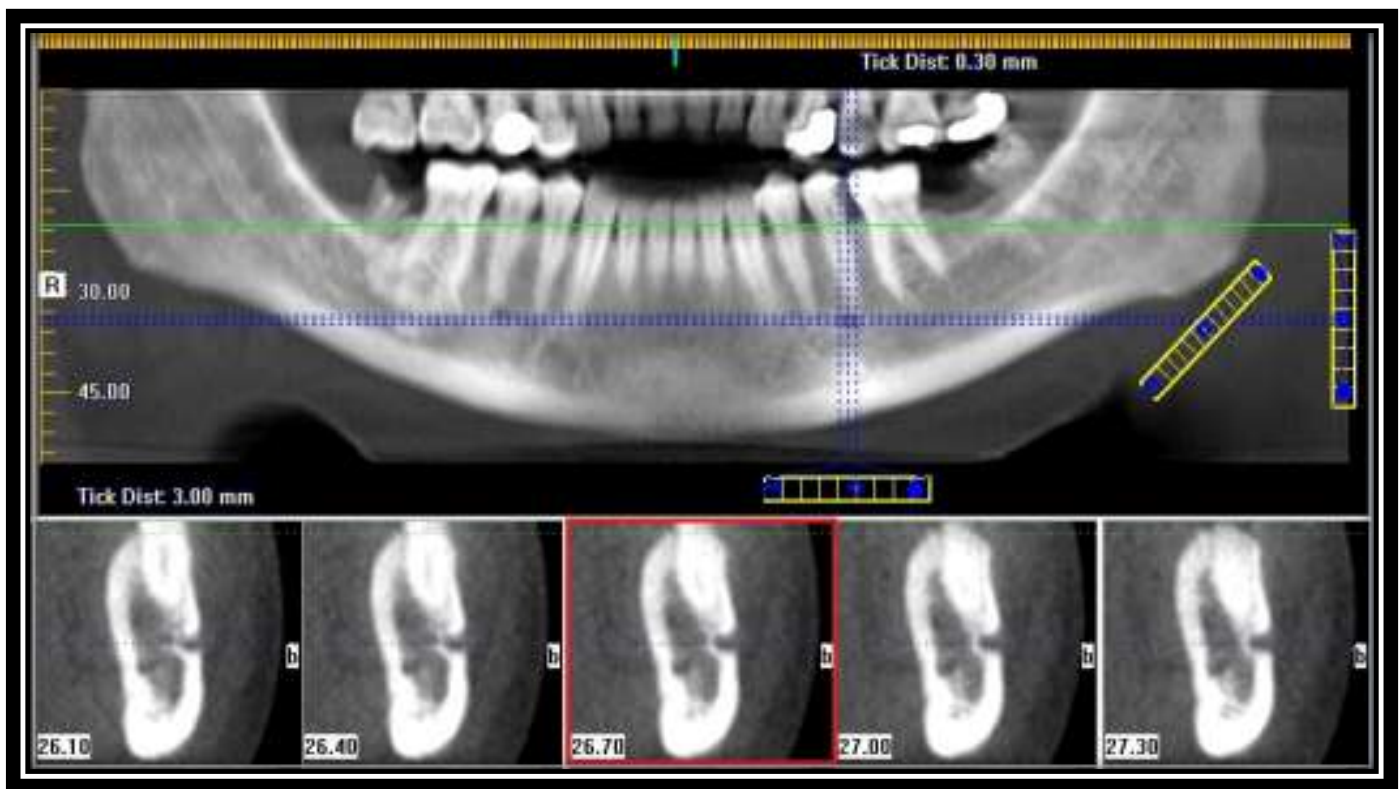

Figure 8: Selection of the coronal cross sectional image on a CBCT scan of one of the investigated volunteers. 


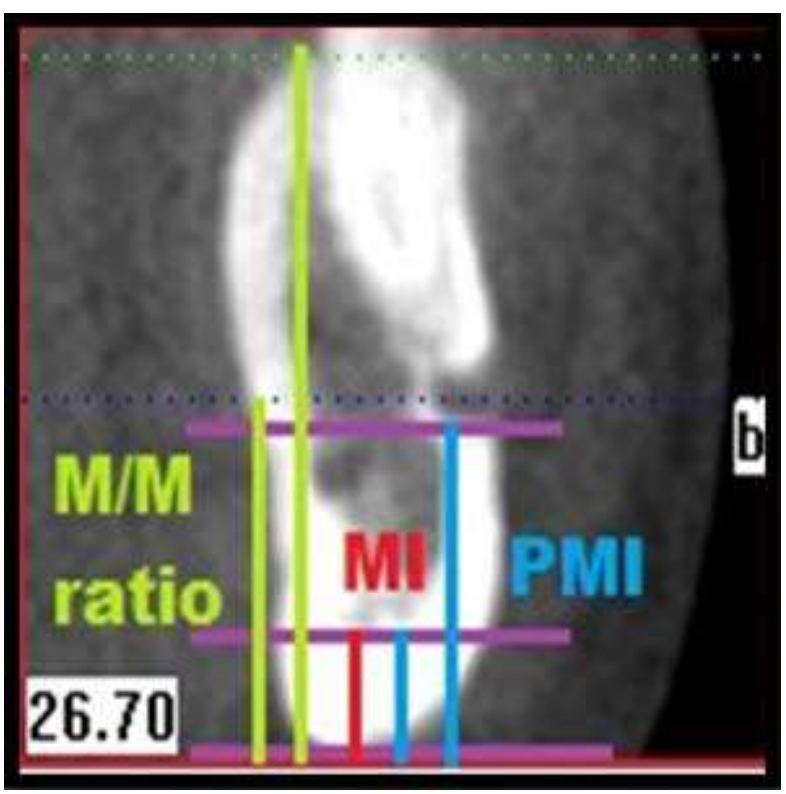

Figure 9: Measurement of the 3 indices (MI, PMI\&M/M ratio) on one of the investigated volunteers on the selected CBCT coronal cross sectional image.

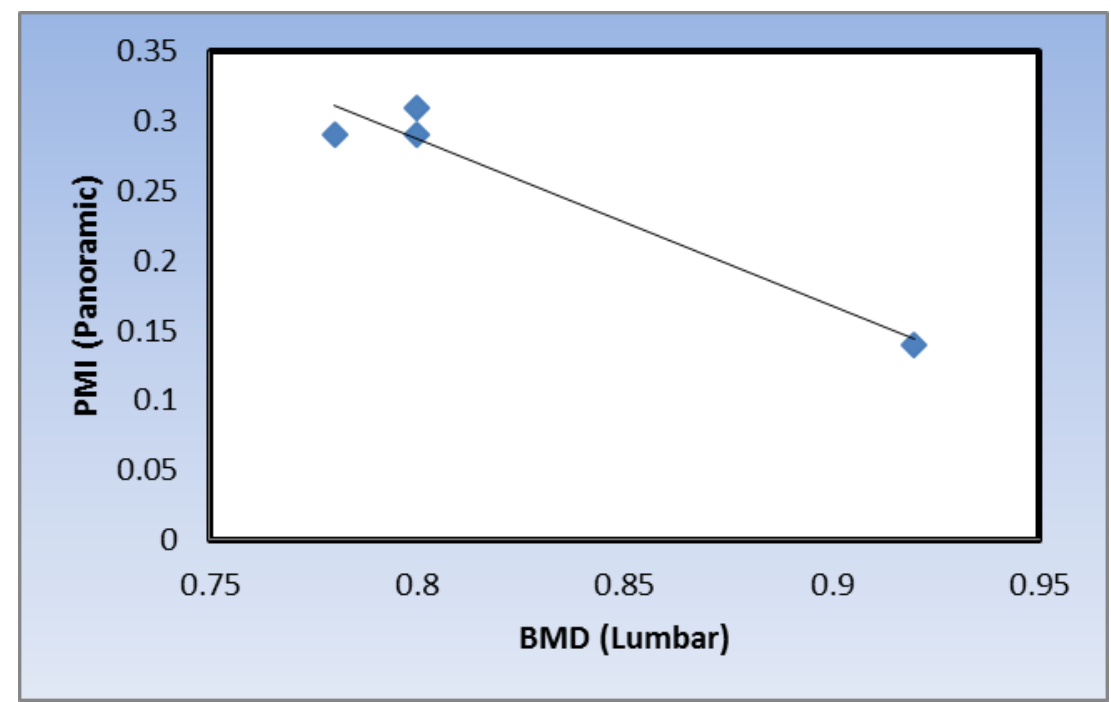

Figure 10: Scatter diagram showing negative (inverse) correlation between PMI (Digital panoramic) and BMD measurements of Lumbar vertebrae in Osteoporotic group. 


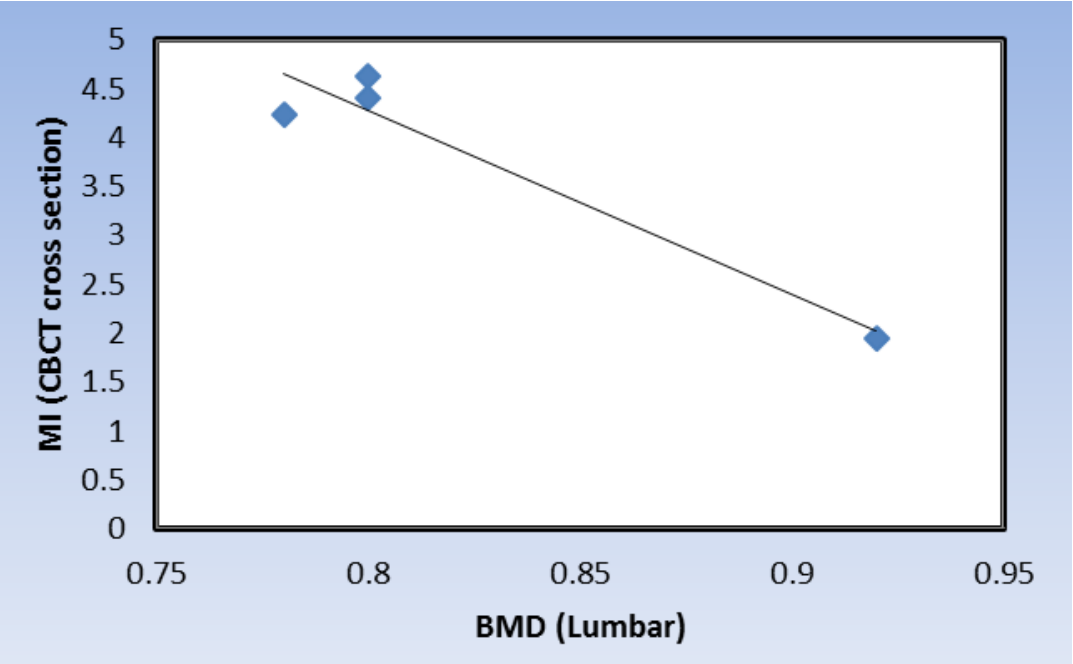

Figure 11: Scatter diagram showing negative (inverse) correlation between MI (CBCT cross-sectional) and BMD measurements of Lumbar vertebrae in Osteoporotic group.

Table 1:- Results of regression analysis.

\begin{tabular}{|c|c|c|}
\hline Measurement & Score & $\boldsymbol{P}$-value \\
\hline MI (Panoramic) & 5.960 & $0.015^{*}$ \\
\hline Ante-gonial index (Panoramic) & 1.833 & 0.176 \\
\hline Gonial index (Panoramic) & 1.584 & 0.208 \\
\hline Gonial angle (Panoramic) & 0.112 & 0.737 \\
\hline PMI (Panoramic) & 7.987 & $0.005^{*}$ \\
\hline M/MR ratio (Panoramic) & 1.286 & 0.257 \\
\hline MI (CBCT Reformatted Panoramic) & 3.936 & $0.047^{*}$ \\
\hline PMI (CBCT Reformatted Panoramic) & 2.393 & 0.122 \\
\hline M/M ratio (CBCT Reformatted Panoramic) & 0.001 & 0.977 \\
\hline MI (CBCT cross section Panoramic) & 5.195 & $0.023^{*}$ \\
\hline PMI (CBCT cross section Panoramic) & 3.289 & 0.070 \\
\hline M/M ratio (CBCT cross section Panoramic) & 1.674 & 0.196 \\
\hline
\end{tabular}

*: Significant at $P \leq 0.05$

\section{Discussion:-}

White, 2002 had stated that research teams worldwide have recently tried to develop methods for using the jaw bones to predict skeletal BMD. According to Çakur et al., 2009, clinicians have started to focus on some mandibular panoramic indices such as mental index (MI) and panoramic mandibular index (PMI) for the BMD assessment. This study focused on the mental index (MI), the panoramic mandibular index (PMI), the alveolar bone resorption degree (M/M ratio), the antegonial index (AI), the gonial angle (GA) and the gonial index (GI) as they are the most commonly used indices in the assessment of BMD and their usage as predictors for mandibular osteoporosis is still controversial. Accordingly, the authors were motivated to use these indices specifically in order to add to this open field of research.

Osteoporosis is a systemic skeletal disease characterized by low bone mass and microarchitectural deterioration of bone tissue, with a consequent increase in bone fragility and susceptibility to fracture. Epidemiological studies have estimated that there would be an exponential increase in the incidence of osteoporotic fractures with the increase of age (Yeap et al., 2012). Early intervention may maximize bone mass retention and enhancement and thus reduce the risk of fracture (Delaney, 2006).

In the current study, on correlating the DEXA lumbar vertebrae results with panoramic radiomorphometric indices in the normal, osteopenic and osteoporotic groups, a nonstatistically significant correlation was found in most of the results. 
Many other studies were performed for testing the accuracy of these indices for predicting osteoporosis by correlating them to the BMD values of lumbar vertebrae. Regarding the MI, the study results agree with Çakur et al., 2010 who found that there was no significant correlation between the MI and vertebral BMD in men with osteoporosis. However, the study results disagree with some studies as those of Devlin and Horner, 2002 who found that MI contributed significantly to the diagnosis of low skeletal BMD of lumbar vertebrae and Mahl et al., 2008 who found that MI could differentiate between patients with osteopenia and osteoporosis on the basic of lumbar BMD. Moreover, Leite et al, 2010 also found a statistically significant positive correlation between MI and DEXA lumbar vertebrae results in their normal and osteoporotic groups.

Regarding the PMI, the study results were in concordance with other studies as those done by Çakur et al., 2010 who found that there was no significant correlation between the PMI and vertebral BMD in men with osteoporosis. In addition to Marandi et al., 2010 who also found no significant correlation between PMI and bone densitometry of lumbar vertebrae. Besides to Damilakis and Vlasiadis, 2011 who found that PMI Indices were not statistically significant for that correlation. However, these results are in contrary to those of Mahl et al., 2008 who found that the PMI could differentiate between patients with osteopenia and osteoporosis on the basic of lumbar BMD.

Regarding the M/M Ratio, the results agree with Damilakis and Vlasiadis, 2011 who found that corresponding differences for $\mathrm{M} / \mathrm{M}$ ratio were not statistically significant with lumbar BMD values.

Regarding the GI, the results are similar to Devlin and Horner, 2002 who found that there was no statistically significant correlation between the GI and the DEXA lumbar vertebrae results in their normal, osteopenic and osteoporotic groups. On the contrary, the results disagree with some studies as those of Mahl et al., 2008 who found that GI evaluated in their study could identify a low bone mass density.

Regarding the AI, the study results are parallel to Devlin and Horner, 2002 who found that there was no statistically significant correlation between AI and the DEXA lumbar vertebrae results in their normal, osteopenic and osteoporotic groups. Besides to Çakur et al., 2010 who found that there was no significant correlation between the $\mathrm{AI}$ and vertebral BMD in men with osteoporosis. On the other hand, the results disagree with those reported by Mahl et al., 2008 who found that AI evaluated in their study could identify a low bone density. Also, Leite et al., 2010 who found a statistically significant positive correlation between AI and DEXA lumbar vertebrae results in their normal and osteoporotic groups.

Regarding the GA, the study results come in agreement with those given by Dutra et al., 2006 who found that the gonial angle did not show differences among the three categories of skeletal bone status (normal, osteopenic and osteoporotic) in their study. In addition to Leite et al., 2010 who found no statistically significant correlation between the gonial angle and DEXA lumbar vertebrae results in their normal and osteoporotic groups. On the contrary, the results disagree with the results reported by Çakur et al., 2011 who found a negatively significant correlation between the gonial angle and vertebral BMD in osteoporotic men.

In this study, upon correlating the DEXA femur results with panoramic radiomorphometric indices in the normal, osteopenic and osteoporotic groups, a nonstatistically significant correlation was found.

Many other studies were performed for testing the accuracy of these indices for predicting osteoporosis by correlating them to the BMD values of Femur. Regarding the MI, the study results agree with Çakur et al., 2010 who found that there was no significant correlation between the MI and femoral BMD in men with osteoporosis. On the other hand, they disagree with some studies as those done by Mahl et al., 2008 who found that MI could differentiate between patients with osteopenia and osteoporosis depending on femur BMD.

Regarding the PMI, these results were in concordance with other studies as those performed by Çakur et al., 2010 who found that there was no significant correlation between the PMI and Femoral BMD in men with osteoporosis. However, these results are contradicting those of Mahl et al., 2008 who found that the PMI could differentiate between patients with osteopenia and osteoporosis depending on Femoral BMD.

Regarding the GI, these results disagree with some studies as those of Mahl et al., 2008 who found that GI evaluated in their study could identify a low bone density. 
Regarding the AI, the study results are similar to Çakur et al., 2010 who found that there was no significant correlation between the AI and Femoral BMD in men with osteoporosis. However, these results are dissimilar to those of Mahl et al., 2008 who found that AI evaluated in their study could identify a low bone density.

Regarding the GA, the results agree with Dutra et al., 2006 who found that the gonial angle did not show differences among the three categories of skeletal bone status (normal, osteopenic and osteoporotic) in their study.

In our study, on correlating the DEXA forearm results with panoramic radiomorphometric indices in the normal, osteopenic and osteoporotic groups, a nonstatistically significant correlation was also found.

Devlin and Horner, 2002 measured the validity of mandibular cortical indices (MI, GI and AI) on panoramic radiographs in the diagnosis of reduced skeletal bone density and found that GI and AI were non-significantly correlated to the $\mathrm{T}$-scores recorded at the forearm measured by single photon absorptiometry. While, the MI in the same study was significantly correlated with the T-scores recorded at the forearm. No previous studies had correlated the DEXA forearm results (BMD and T-score) with any panoramic radiomorphometric index to be compared to our results.

In our study, on correlating the DEXA lumbar vertebrae, femur and forearm results with CBCT reformatted panoramic view indices in the normal, osteopenic and osteoporotic groups, a nonstatistically significant correlation was found. No previous studies were performed correlating the CBCT reformatted panoramic view indices and Tscore of lumbar vertebrae, femur and forearm to be compared with this study results. Similarly, on correlating the DEXA lumbar vertebrae and femur results with CBCT cross section view indices in the normal, osteopenic and osteoporotic groups, a nonstatistically significant correlation was found in most of the results.

Comparable to the current study results, Koh and Kim, 2011 found that there was no correlation between the normal and osteoporotic groups in the CTI (I) (PMI) during evaluating the potential use of the computed tomography indices (CTI) on CBCT images by correlating it with the lumbar and femoral BMD in postmenopausal women. On the contrary, they found that there were significant differences between the groups in the CTMI (MI).

In the sudy results, on correlating the DEXA forearm results with CBCT cross section view indices in the normal, osteopenic and osteoporotic groups, a nonstatistically significant correlation was found. No previous studies had correlated the DEXA forearm results (BMD and T-score) with any CBCT cross section view index to be compared to our results.

Further agreements to our results; include a study by Gulsahi et al., 2010 concluded that the BMD of the jaws was not correlated with femoral BMD. In addition to Çakur et al., 2009 who did not find any correlation between the BMD obtained in the densitometry of the jaw and that obtained in the hip and lumbar spine. Contradicting our results, Devlin and Horner, 2002 found a relationship between BMD of the mandible and the forearm BMD.

To further confirm our results, logistic regression model was used to determine the most predictive index for osteoporosis. The results showed that the significant predictors of osteoporosis are the following variables arranged from the highest to lowest scores: PMI (yielded from panoramic views) followed by MI (yielded from panoramic views), then MI (yielded from CBCT cross section) and finally MI (yielded from CBCT reformatted panoramic views). These results indicate that PMI and MI, with a larger sample size, may serve as prognostic osteoporosis indices and may be useful in determining the mandibular bone mineral status. Furthermore, the MI proved to be a good predictor whether on panoramic or CBCT views as being simple; needing no further calculations and away from the dental status or alveolar bone height.

\section{Conclusion:-}

Within the limitation of the current study regarding the limited sample size, the bone of the mandible does not appear to reflect the characteristics of the skeleton as a whole. PMI and MI, with a larger sample size, may serve as prognostic osteoporosis indices. 


\section{Acknowledgment:-}

The authors acknowledge the Rheumatology Department, Faculty of Medicine, Cairo University, for the constant help through this work.

\section{Duality of Interest:-}

No potential conflicts of interest relevant to this study were reported.

\section{Funding:-}

This research did not receive any specific grant from funding agencies in the public, commercial, or not-for-profit sectors.

\section{References:-}

1. Benson BW, Prihoda TJ and Glass BJ (1991): Variations in adult cortical bone mass as measured by a panoramic mandibular index. Oral Surg Oral Med Oral Pathol., 71(3):617-21.

2. Berco M, Rigali P H, Matthew Miner R, Deluca S, Andreson NK and Will LA (2009): Accuracy and reliability of linear cephalometric measurements from cone-beam computed tomography scans of a dry human skull. Am J Orthod Dentofacial Orthop., 136(1):17.e1-9.

3. Bras J, Van Ooij CP, Abraham-Inpijn L, Kusen GJ and Wilmink JM (1982): Radiographic interpretation of the mandibular angular cortex: a diagnostic tool in metabolic bone loss. Part I. Normal state. Oral Surg Oral Med Oral Pathol., 53(5):541-5.

4. Çakur B, Dağistan S and Sümbüllü MA (2010): No correlation between mandibular and nonmandibular measurements in osteoporotic men. Acta Radiol., 51(7):789-792.

5. Çakur B, Dağistan S, Harorli A and Ezmeci EB (2011): The mandibular angle in osteoporotic men. Med Oral Pathol Oral Cir Bucal., 1; 16(2):e181-4.

6. Çakur B, Dagistan S, Sahin A, Harorli A and Yilmaz A (2009): Reliability of mandibular cortical index and mandibular bone mineral density in the detection of osteoporotic women. Dentomaxillofac Radiol., 38(5):25561.

7. Damilakis J and Vlasiadis K (2011): Have panoramic indices the power to identify women with low BMD at the axial skeleton?. Physica Medica., 27(1): 39-43.

8. Delaney MF (2006): Strategies for the prevention and treatment of osteoporosis during early post menopause. American Journal of Obstetrics and Gynecology., 194(2 suppl): S12-23.

9. Devlin H and Horner K (2002): Mandibular radiomorphometric indices in the diagnosis of reduced skeletal bone mineral density. Osteopor Int., 13(5):373-8.

10. Dutra V, Devlin H, Susin C, Yang J, Horner K and Fernandes AR (2006): Mandibular morphological changes in low bone mass edentulous females: evaluation of panoramic radiographs. Oral Surg Oral Med Oral Pathol Oral Radiol Endod., 102(5):663-8.

11. Dutra V, Yang J, Devlin H and Susin Ch (2005): Radiomorphometric indices and their relation to gender, age, and dental status. Oral Surg Oral Med Oral Pathol Oral Radiol Endod., 99(4):479-84.

12. Gulsahi A, Paksoy CS, Ozden S, Kucuk NO, Cebeci AR and Genc Y (2010): Assessment of Bone Mineral Density in The Jaws and Its Relationship to radiomorphometric Indices. Dentomaxillofac Radiol., 39(5):284-89.

13. Hirai T, Ishijima T, Hashikawa Y and Yajima T (1993): Osteoporosis and reduction of residual ridge in edentulous patients. J Prosthet Dent., 69(1): 49-56.

14. Kazakia GJ and Majumdar S (2006): New imaging technologies in the diagnosis of osteoporosis. Rev Endocr Metab Disord., 7(1-2):67-74.

15. Klemetti E and Vainio MS (1993): Effect of bone mineral density in skeleton and mandible on extraction of teeth and clinical alveolar height. J Prosthet Dent., 70(1): 21-25.

16. Klemetti E, Kolmakov S and Kroger H (1994): Pantomography in assessment of the osteoporosis risk group. Scand J Dent Res., 102(1):68-72.

17. Koh KJ and Kim KA (2011): Utility of the computed tomography indices on cone beam computed tomography images in the diagnosis of osteoporosis in women. Imaging Science in Dentistry., 41(3):101-6.

18. Ledgerton D, Horner K Devlin H and Worthington H (1997): Panoramic mandibular index as a radiomorphometric tool an assessment of precision. Dentomaxillofac Radiol., 26(2):95-100.

19. Ledgerton D, Horner K, Devlin H and Worthington H (1999): Radiomerphometric indices of the mandible in a British female population. Dentomaxillofac Radiol., 28(3):173-81. 
20. Leite AF, Figueiredo PT, Guia CM, Melo NS and de Paula AP (2010): Correlations between seven panoramic radiomorphometric indices and bone mineral density in postmenopausal women. Oral Surg Oral Med Oral Pathol Oral Radiol Endod., 109(3):449-456.

21. Mahl CRW, Licks R and Fontanella VRC (2008): Comparison of morphometric indices obtained from dental panoramic radiography for identifying individuals with osteoporosis/osteopenia. Radiol Bras., 41(3):183-187.

22. Marandi S, Bagherpour A, Imanimoghaddam M, Hatef MR and Haghighi AR (2010): Panoramic-Based Mandibular Indices and Bone Mineral Density of Femoral Neck and Lumbar Vertebrae in Women. J Dent (Tehran)., 7(2):98-106.

23. Nakamoto T, Taguchi A, Ohtsuka M, Suei Y, Fujita M, Tanimoto K, Tsuda M, Sanada M, Ohama K, Takahashi J and Rohlin M (2003): Dental panoramic radiograph as a tool to detect postmenopausal women with low bone mineral density: untrained general dental practitioners' diagnostic performance. Osteoporos Int., 14(8): 659664.

24. Nakamura Y, Suzuki T, Kamimura M, Ikegami S, Uchiyama S and Kato H (2017): Alfacalcidol Increases the Therapeutic Efficacy of Ibandronate on Bone Mineral Density in Japanese Women with Primary Osteoporosis. Tohoku J Exp Med., 241(4):319-326.

25. Scarfe WC, Farman AG and Sukovic P (2006): Clinical applications of cone-beam computed tomography in dental practice. J Can Dent Assoc., 72(1): 75-80.

26. White SC (2002): Oral radiographic predictors of osteoporosis. Dentomaxillofacial Radiology., 31(2): 84-92.

27. Xie QF and Ainamo A (2004): Correlation of gonial angle size with cortical thickness, height of the mandibular residual body, and duration of edentulism. J Prosthet Dent., 91(5):477-82.

28. Yeap SS, Hew FL, Lee JK, Goh EML, Chee W, Mumtaz M, Damodaran P, Lim HH and Chan SP (2013): The Malaysian Clinical Guidance On The Management Of Postmenopausal Osteoporosis, 2012: A Summary. Int J Rheum Dis., 16(1):30-40.

29. Zarch SH, Bagherpour A, Langaroodi AJ, Yazdi AA and Safaei A (2011): Evaluation of the Accuracy of Panoramic Radiography in Linear Measurements of the Jaws. Iran J Radiol., 8(2): 97-102. 